[Article]

\title{
基于咔唑的双光子荧光次氯酸根探针光学性质及响应机理
}

\author{
王 昕 张玉瑾 王传奎* \\ (山东师范大学物理与电子科学学院, 济南 250014)
}

\begin{abstract}
摘要: 采用含时密度泛函理论与响应函数理论相结合的方法, 研究了两种实验室新合成的水溶性双光子苂光 次氯酸根 $\left(\mathrm{ClO}^{-}\right)$探针分子 $\mathrm{HCH}$ 和 $\mathrm{HCM}$ 的单光子吸收、双光子吸收和荧光发射性质。计算结果表明, $\mathrm{HCH}$ 和 $\mathrm{HCM}$ 分子与 $\mathrm{ClO}^{-}$反应后, 生成物的光吸收和光发射性质均发生明显变化, 相应的吸收和发射峰位都发生了 明显蓝移, 苂光强度展现出明显的增强。另外, 两探针分子都具有较大的双光子吸收截面, 且与 $\mathrm{ClO}^{-}$反应 后, 生成物的双光子吸收截面值显著增加, 因此两分子均可作为性能优良的双光子苂光探针分子。此外, 通 过分析 $\mathrm{HCH}$ 和 $\mathrm{HCM}$ 分子与 $\mathrm{ClO}^{-}$反应前后的 Mulliken 电荷布居情况, 从理论上证实了该系列苂光探针分子的 识别机理是 $\mathrm{C}=\mathrm{N}$ 异构化。
\end{abstract}

关键词: 荧光探针; 双光子吸收; 次氯酸根; $\mathrm{C}=\mathrm{N}$ 异构化

中图分类号：0644；0641

\section{Optical Properties and Responsive Mechanism of Carbazole-Based Two-Photon Fluorescent Probes for the Detection of Hypochlorite}

\author{
WANG Xin ZHANG Yu-Jin WANG Chuan-Kui* \\ (School of Physics and Electronics, Shandong Normal University, Jinan 250014, P. R. China)
}

\begin{abstract}
The one- and two-photon absorption and emission properties of the two newly synthesized watersoluble two-photon fluorescent probes $\left(\mathrm{HCH}\right.$ and $\mathrm{HCM}$ ) for the detection of $\mathrm{ClO}^{-}$were investigated using timedependent density functional theory in combination with response theory. Dramatic changes in the photophysical properties of the molecules following reaction with $\mathrm{ClO}^{-}$were predicted. The photoabsorption and photoemission peaks for both compounds were clearly blue shifted, while the emission intensities were enhanced. Both probes were also found to have large two-photon cross sections. Importantly, in the presence of $\mathrm{ClO}^{-}$the two-photon cross section of the molecules increased significantly, which indicated that both $\mathrm{HCH}$ and $\mathrm{HCM}$ should be good two-photon fluorescent probes for sensing $\mathrm{ClO}^{-}$. The responsive mechanism of the probes was explored by analyzing the molecular Mulliken charge population, which was attributed to $\mathrm{C}=\mathrm{N}$ isomerization.
\end{abstract}

Key Words: Fluorescent probe; Two-photon absorption; $\mathrm{ClO}^{-} ; \mathrm{C}=\mathrm{N}$ isomerization

\section{1 引言}

作为人体内重要的活性氧之一, 次氯酸根 $\left(\mathrm{ClO}^{-}\right)$直接参与生命体众多的生理过程, 在生命活 动中发挥着至关重要的作用 ${ }^{1-4}$ 。一方面, 次氯酸
根能够维持细胞内的氧化还原平衡状态, 同时在 免疫系统中展现出强大的抗菌能力5, 另一方面, 过量的次氯酸根又会导致组织损伤, 并且引发一 系列疾病, 例如动脉硬化症、关节炎等 ${ }^{7-9}$ 。因

Received: June 6, 2016; Revised: September 30, 2016; Published online: September 30, 2016.

${ }^{*}$ Corresponding author. Email: ckwang@sdnu.edu.cn; Tel: +86-531-86180892.

The project was supported by the National Natural Science Foundation of China (11404193), Natural Science Foundation of Shandong Province,

China (ZR2014AM026), and Taishan Scholar Project of Shandong Province, China.

国家自然科学基金(11404193), 山东省自然科学基金(ZR2014AM026)和山东省泰山学者资助项目

(C) Editorial office of Acta Physico-Chimica Sinica 
此, 对次氯酸根的探测成为一个重要的研究课 题。在诸多的探测方法中, 苂光显微法由于具有 高效、灵敏等一系列优势而被广泛采用。

近年来, 开发新型次氯酸根苂光探针越来越 受到人们的关注。Cheng 等 ${ }^{10}$ 设计合成了基于氟硼 吡咯的 $\mathrm{HClO}$ 探针, 该探针分子的三苯基磷基团能 够靶向活细胞的线粒体, 实验结果显示, 该探针 分子能够对次氯酸实现特异性识别, 随着次氯酸 的滴定, 溶液展现出肉眼可区分的颜色转变。Xiao 等 ${ }^{11}$ 以吩噻嗪基团作为苂光团, 以季铵化吡啶部分 作为线粒体的靶向基团, 设计合成了 $\mathrm{ClO}^{-}$苂光探 针 PZ-Py。并利用该探针实现了对巨噬细胞和活体 细胞中内源性 $\mathrm{ClO}^{-}$的苂光成像检测。然而, 之前 所报道的多数次氯酸根探针分子, 包括以上提到 的两探针, 都是基于单光子激发的荧光探针分 子。单光子荧光显微技术由于短波激发限制了其 在深层成像中的应用, 并伴有光损伤、光漂白等 危害。近几年来, 双光子荧光显微因其高选择性 和灵敏度, 能够实时检测, 数据采集简单快捷, 时空分辨率高等优点而被广泛应用 ${ }^{12-18}$ 。

近日, $\mathrm{Li}$ 等 ${ }^{19}$ 在实验上合成了基于咔唑的水溶 性双光子苂光探针分子 $\mathrm{HCH}$ 和 $\mathrm{HCM}$, 两探针分子 均以肪基团作为 $\mathrm{ClO}^{-}$的识别基团, 利用该系列探 针分子能够实现长波激发下, 对 $\mathrm{ClO}^{-}$的快速响 应、高选择性识别以及活细胞中 $\mathrm{ClO}^{-}$的检测和生 物成像。尽管实验测量结果显示 $\mathrm{HCH}$ 和 $\mathrm{HCM}$ 探针 分子展现出良好的应用前景, 但目前两探针分子 的响应机理尚未得到充分的理论研究。另外, 虽 然末端烷基基团对探针分子探测性能的影响在实 验工作中得到了讨论, 但是仍然缺乏相关的理论 研究。本文中, 采用含时密度泛函理论(TDDFT)和 响应理论相结合的方法, 系统地研究了实验室合 成的双光子荧光探针分子 $\mathrm{HCH}$ 和 $\mathrm{HCM}$ 与 $\mathrm{ClO}^{-}$反 应前后的单、双光子吸收和荧光发射性质, 并在 此基础上通过分析分子体系电荷布居探究了其响 应机理。

\section{2 计算方法}

本文所使用的理论方法参见文献 ${ }^{20}$, 这里仅给 出计算细节。在 B 3 LYP 水平上优化了分子的基态 几何结构, 基于优化得到的分子基态几何结构, 在密度泛函理论(DFT)/CAM-B3LYP 水平上采用响 应理论方法计算了分子的单光子吸收性质, 包括
激发能、激发波长、振子强度和跃迁特性。基于 相同的分子基态几何结构, 在 DFT/B3LYP 水平上 计算了分子最低八个激发态的双光子吸收性质, 包括激发能、对应的双光子吸收波长和双光子吸 收截面。在含时密度泛函理论/B3LYP 水平上优化 了分子第一激发态的几何结构并得到了分子的荧 光发射性质。其中, 分子结构优化是在 Gaussian 09 程序包 ${ }^{21}$ 上进行的, 同时为了确保优化后结构的 稳定性, 我们在相同的计算水平上进行了频率计 算; 分子单光子吸收、苂光发射性质的计算是在 Gaussian 09 程序包上进行的, 双光子吸收性质的 计算是在 Dalton 2013.3 程序包 ${ }^{22}$ 上进行的。以上计 算均选用了 6-31g**基组, 并采用极化连续模型 $(\mathrm{PCM})$ 来描述周围的溶剂环境水 $\left(\mathrm{H}_{2} \mathrm{O}\right)$ 和二甲亚砜 (DMSO) 对分子光学性质的影响。为了直观地展现 分子电荷分布, 我们利用 Gauss View 5.0 做出了分 子的静电势(MEPs)图。

\section{3 结果与讨论}

\section{1 分子结构}

荧光探针分子 $\mathrm{HCH}$ 和 $\mathrm{HCM}$ 的几何结构如图 1 所示, 两分子都以肜基团作为 $\mathrm{ClO}^{-}$的识别基团, 而其吡啶所连的末端基团分别为己基和甲基。探 针分子 $\mathrm{HCH}$ 和 $\mathrm{HCM}$ 与 $\mathrm{ClO}^{-}$反应后, 肜基团发生 氧化脱氢作用生成氧化腈, 碳氮双键被氧化成碳 氮三键, 对应的生成物分别为 $\mathrm{HCH}-\mathrm{CNO}$ 分子和 $\mathrm{HCM}-\mathrm{CNO}$ 分子。

表 1 给出了水溶液中, 分子优化后的部分键长 和二面角情况。 $\mathrm{HCH}$ 分子的 $\mathrm{C}_{1}-\mathrm{N}_{2}$ 键长为 0.149 $\mathrm{nm}$, 将 $\mathrm{HCH}$ 分子吡啶所连的末端基团己基替换成 甲基, 即在 $\mathrm{HCM}$ 分子中, $\mathrm{C}_{1}-\mathrm{N}_{2}$ 键长缩短为 $0.148 \mathrm{~nm}$ 。当两探针分子分别与 $\mathrm{ClO}^{-}$反应后, $\mathrm{C}_{10}-\mathrm{C}_{11} 、 \mathrm{C}_{11}=/ \equiv \mathrm{N}_{12} 、 \mathrm{~N}_{12}-\mathrm{O}_{13}$ 的键长分别由 $0.147 、 0.128 、 0.139 \mathrm{~nm}$ 缩短为 $0.143 、 0.117$ 、 $0.123 \mathrm{~nm}$, 这是由于 $\mathrm{ClO}^{-}$使末端肜基团发生氧化脱 氢作用引起结构上的变化而造成的。探针分子 $\mathrm{HCH}$ 和 $\mathrm{HCM}$ 的吡啶平面与咔唑平面间的二面角分 别为 $179.564^{\circ}$ 和 $179.428^{\circ}$, 与 $\mathrm{ClO}^{-}$反应后, 对应二 面角分别为 $179.821^{\circ}$ 和 $179.652^{\circ}$ 。可见两探针分子 均具有较好的平面性, 并且与被探测底物作用以 后, 分子体系的平面性得到了增强, 另外与 $\mathrm{HCM}$ 分子和 $\mathrm{HCM}-\mathrm{CNO}$ 分子相比, $\mathrm{HCH}$ 分子和 $\mathrm{HCH}-$ $\mathrm{CNO}$ 分子对应的平面性更好一些, 这有利于分子 


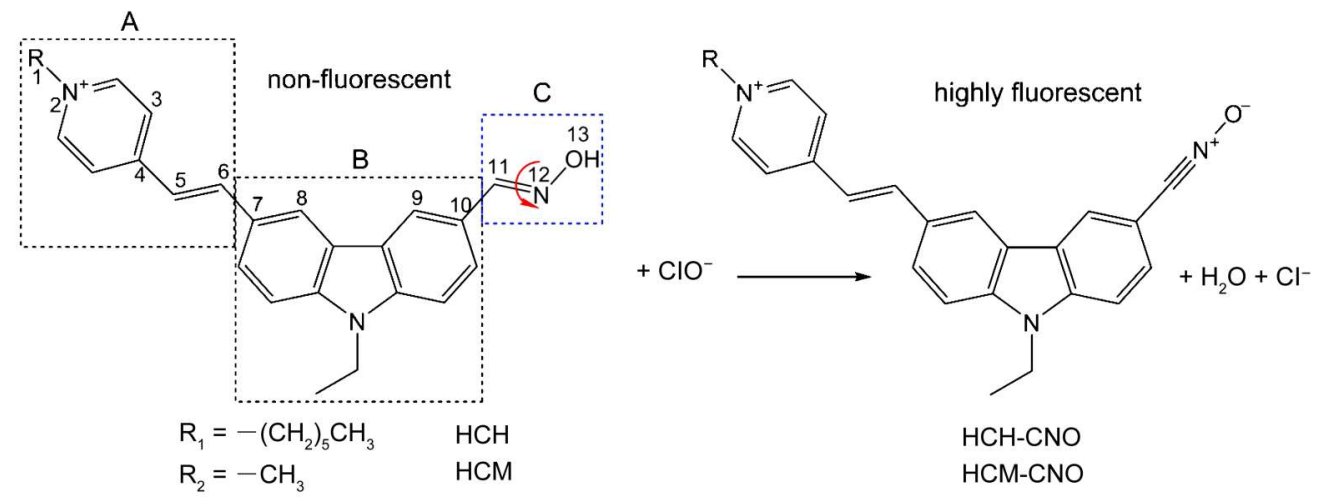

图 1 探针分子 $\mathrm{HCH}, \mathrm{HCM}$ 的几何结构示意图及与 $\mathrm{ClO}^{-}$反应过程

Fig.1 Geometric structures of probe molecules $\mathrm{HCH}, \mathrm{HCM}$ and reaction process for sensing $\mathrm{ClO}^{-}$

表 1 水中分子优化后的部分键长和二面角

Table 1 Selective bond lengths and dihedral angles of the optimized compounds in $\mathrm{H}_{2} \mathrm{O}$

\begin{tabular}{|c|c|c|c|c|c|}
\hline \multirow{2}{*}{ Molecule } & \multicolumn{4}{|c|}{ Bond length/nm } & \multirow{2}{*}{$\angle \mathrm{C}_{4} \mathrm{C}_{5} \mathrm{C}_{6} \mathrm{C}_{7} /\left({ }^{\circ}\right)$} \\
\hline & $\mathrm{C}_{1}-\mathrm{N}_{2}$ & $\mathrm{C}_{10}-\mathrm{C}_{11}$ & $\mathrm{C}_{11}=/ \equiv \mathrm{N}_{12}$ & $\mathrm{~N}_{12}-\mathrm{O}_{13}$ & \\
\hline $\mathrm{HCH}$ & 0.149 & 0.147 & 0.128 & 0.139 & 179.564 \\
\hline $\mathrm{HCM}$ & 0.148 & 0.147 & 0.128 & 0.139 & 179.428 \\
\hline $\mathrm{HCH}-\mathrm{CNO}$ & 0.149 & 0.143 & 0.117 & 0.123 & 179.821 \\
\hline $\mathrm{HCM}-\mathrm{CNO}$ & 0.148 & 0.143 & 0.117 & 0.123 & 179.652 \\
\hline
\end{tabular}

内电荷转移的发生, 因此推测 $\mathrm{HCH}$ 分子具有更强 的光吸收性质。

\section{2 单光子吸收性质}

表 2 给出了在水溶液和 DMSO 溶液中, 探针分 子与 $\mathrm{ClO}^{-}$反应前后的最大单光子吸收波长及相应 的激发能、振子强度和跃迁特性。计算结果表

表 2 分子在低能量范围内的单光子吸收性质, 包括激发能 $\left(\boldsymbol{E}_{\mathrm{opa}}\right)$ 、对应的吸收波长 $\left(\lambda_{\mathrm{opa}}\right)$ 、振子强度 $\left(\boldsymbol{\delta}_{\mathrm{opa}}\right)$ 和跃迁特性

Table 2 One-photon absorption properties of the compounds in lower energy region, including the excitation energy $\left(E_{\text {opa }}\right)$, the corresponding absorption wavelength $\left(\lambda_{\text {opa }}\right)$, the oscillator strength $\left(\delta_{\text {opa }}\right)$, and the transition feature

\begin{tabular}{ccccc}
\hline Molecule & $E_{\text {opa }} / \mathrm{eV}$ & $\lambda_{\text {opa }} / \mathrm{nm}$ & $\delta_{\text {opa }} /($ a.u. $)$ & Transition feature \\
\hline HCH & 3.12 & $398^{\mathrm{a}}$ & 1.38 & $S_{0} \rightarrow S_{1}, \mathrm{H} \rightarrow \mathrm{L}, 81.2 \%$ \\
& 3.09 & $401^{\mathrm{b}}$ & 1.40 & $S_{0} \rightarrow S_{1}, \mathrm{H} \rightarrow \mathrm{L}, 80.8 \%$ \\
& & $422^{\mathrm{c}}$ & & \\
$\mathrm{HCH}-\mathrm{CNO}$ & 3.18 & $390^{\mathrm{a}}$ & 1.42 & $S_{0} \rightarrow S_{1}, \mathrm{H} \rightarrow \mathrm{L}, 80.1 \%$ \\
& 3.15 & $393^{\mathrm{b}}$ & 1.44 & $S_{0} \rightarrow S_{1}, \mathrm{H} \rightarrow \mathrm{L}, 79.6 \%$ \\
$\mathrm{HCM}$ & 3.12 & $416^{\mathrm{c}}$ & & \\
& 3.10 & $407^{\mathrm{a}}$ & 1.30 & $S_{0} \rightarrow S_{1}, \mathrm{H} \rightarrow \mathrm{L}, 81.5 \%$ \\
& & $419^{\mathrm{c}}$ & & \\
$\mathrm{HCM}-\mathrm{CNO}$ & 3.18 & $389^{\mathrm{a}}$ & 1.35 & $S_{0} \rightarrow S_{1}, \mathrm{H} \rightarrow \mathrm{L}, 80.3 \%$ \\
& 3.16 & $392^{\mathrm{b}}$ & 1.37 & $S_{0} \rightarrow S_{1}, \mathrm{H} \rightarrow \mathrm{L}, 79.8 \%$ \\
& & $413^{\mathrm{c}}$ & & \\
\hline
\end{tabular}

${ }^{\mathrm{a}}$ in $\mathrm{H}_{2} \mathrm{O}$, ${ }^{\mathrm{b}}$ in dimethylsulfoxide (DMSO), ${ }^{\mathrm{c}}$ experimental results ${ }^{19}$; $\mathrm{H}$ (L) denotes the HOMO (LUMO).
明，在低能量范围内，分子 HCH、HCH-CNO、 $\mathrm{HCM} 、 \mathrm{HCM}-\mathrm{CNO}$ 在水溶液中的最大单光子吸收 波长分别位于 398、390、397、389 nm 处, 其对应 的振子强度分别为 $1.38 、 1.42 、 1.30 、 1.35$ a.u., 在 DMSO 溶液中分子的最大单光子吸收波长分别位 于 401、393、400、392 nm 处, 其对应的振子强度 分别为 $1.40 、 1.44 、 1.33 、 1.37$ a.u.。可见, 与在水 溶液中的单光子吸收相比, 分子在 DMSO 溶液中 的最大单光子吸收波长发生了微小红移, 并且振 子强度增强。另外, 两探针分子与 $\mathrm{ClO}^{-}$反应后, 最大单光子吸收峰位置均出现了蓝移, 这与实验 测量结果在趋势上是一致的, 且在 DMSO 溶液中 计算所得结果比在水溶液中计算所得结果与实验 测量值符合得更好。

探针与被检测物 $\mathrm{ClO}^{-}$反应后, 识别基团弜中 的碳氮双键被 $\mathrm{ClO}^{-}$氧化为碳氮三键, 且末端基团 由给电子能力较弱的 $-\mathrm{OH}$ 变为给电子能力较强的 $-\mathrm{O}^{-}$。这使得分子体系中电子供体和电子受体的 推拉电子能力发生了变化, 进一步影响了分子体 系光吸收性质的变化。为了更好地理解被检测物 $\mathrm{ClO}^{-}$影响分子光吸收的内在物理机制, 我们结合 分子轨道分布情况对光吸收过程进行了分析。第 一性原理计算的结果显示, 分子 $\mathrm{HCH} 、 \mathrm{HCH}-$ $\mathrm{CNO} 、 \mathrm{HCM} 、 \mathrm{HCM}-\mathrm{CNO}$ 在水溶液中的最大单光 
子吸收态均位于第一激发态, 主要由最高占据分 子轨道(HOMO)到最低未占据分子轨道(LUMO)的 跃迁贡献, 该跃迁对电子态贡献的比例分别为 $81.2 \% 、 80.1 \% 、 81.5 \% 、 80.3 \%$ 。表3 给出了各分 子前线轨道能量以及 HOMO 和 LUMO 之间的能隙 $\left(\Delta E_{\mathrm{H}-\mathrm{L}}\right)$ 。与 $\mathrm{ClO}^{-}$反应后两探针分子的 $\mathrm{HOMO}$ 能级 与反应前相比稍有下降, 而 LUMO 能级能量不 变, 导致 HOMO 和 LUMO 间的能隙由 $0.10 \mathrm{eV}$ 增加 到 $0.11 \mathrm{eV}$, 因此反应后分子的最大单光子吸收峰 出现蓝移。探针 $\mathrm{HCH}$ 和 $\mathrm{HCM}$ 分子的各前线轨道能 量以及 HOMO 到 LUMO 的能隙值均相同, 可以看 出末端烷基基团对分子体系的前线轨道分布情况 影响不大。

\section{3 双光子吸收性质}

表 4 给出了在 DMSO 溶液中分子最低八个激发 态对应的激发能、双光子吸收波长和双光子吸收 截面。在低能量范围内, 两探针分子 $\mathrm{HCH} 、 \mathrm{HCM}$
的最大双光子吸收均发生在第六激发态, 其最大 双光子吸收截面分别为 $1421 、 1207 \mathrm{GM}$ ，对应的 双光子吸收峰位于 $618 、 621 \mathrm{~nm}$ 处。与 $\mathrm{ClO}^{-}$反应 后, 生成物 HCH-CNO、HCM-CNO 分子的最大双 光子吸收均发生在第八激发态, 其最大双光子吸 收截面分别为 $2824 、 2427 \mathrm{GM}$ ，对应的双光子吸 收峰位于 600、603 nm 处。两探针分子 $\mathrm{HCH}$ 、 $\mathrm{HCM}$ 在与 $\mathrm{ClO}^{-}$反应后, 最大双光子吸收波长均蓝 移了 $18 \mathrm{~nm}$, 最大双光子吸收截面值显著增加, 分 别增加了 1403、1220 GM。值得注意的是，与 $\mathrm{HCM}-\mathrm{CNO}$ 分子相比, $\mathrm{HCH}-\mathrm{CNO}$ 分子的最大双光 子吸收截面较大, 这与实验测量结果定性一致 ${ }^{19}$ 。

表 5 给出了所研究的分子体系在水溶液中的双 光子吸收性质, 从表中可以看到, 在水溶液中 $\mathrm{HCH}$ 和 $\mathrm{HCM}$ 分子的最大双光子吸收截面分别为 1479、1265 GM, 对应的双光子吸收波长分别位于 623、621 nm 处, 而加入 $\mathrm{ClO}^{-}$以后, 分子体系的最

表 3 水中分子的前线轨道能量

Table 3 Frontier orbital energies of the compounds in $\mathrm{H}_{2} \mathrm{O}$

\begin{tabular}{|c|c|c|c|c|c|c|c|c|c|}
\hline \multirow{2}{*}{ Molecule } & \multicolumn{8}{|c|}{ Energy/eV } & \multirow{2}{*}{$\Delta E_{\mathrm{H}-\mathrm{L}} / \mathrm{eV}$} \\
\hline & $\mathrm{H}-3$ & $\mathrm{H}-2$ & $\mathrm{H}-1$ & $\mathrm{H}$ & $\mathrm{L}$ & $\mathrm{L}+1$ & $\mathrm{~L}+2$ & $\mathrm{~L}+3$ & \\
\hline $\mathrm{HCH}$ & -0.27 & -0.25 & -0.22 & -0.20 & -0.10 & -0.05 & -0.05 & -0.04 & 0.10 \\
\hline $\mathrm{HCH}-\mathrm{CNO}$ & -0.26 & -0.25 & -0.23 & -0.21 & -0.10 & -0.05 & -0.05 & -0.04 & 0.11 \\
\hline $\mathrm{HCM}$ & -0.27 & -0.25 & -0.22 & -0.20 & -0.10 & -0.05 & -0.05 & -0.04 & 0.10 \\
\hline $\mathrm{HCM}-\mathrm{CNO}$ & -0.26 & -0.25 & -0.23 & -0.21 & -0.10 & -0.05 & -0.05 & -0.04 & 0.11 \\
\hline
\end{tabular}

表 4 分子在 DMSO 溶液中最低八个激发态的激发能 $\left(E_{\mathrm{tpa}}\right)$ 、对应的双光子吸收波长 $\left(\lambda_{\mathrm{tpa}}\right)$ 以及双光子吸收截面 $\left(\sigma_{\mathrm{tpa}}\right)$

Table 4 Excitation energy $\left(E_{\mathrm{tpa}}\right)$, the corresponding two-photon absorption (TPA) wavelength $\left(\lambda_{\mathrm{tpa}}\right)$, and the TPA cross section $\left(\sigma_{\mathrm{tpa}}\right)$ of the lowest eight excited states for the compounds in DMSO solvent

\begin{tabular}{|c|c|c|c|c|c|c|c|}
\hline Molecule & $E_{\mathrm{tpa}} / \mathrm{eV}$ & $\lambda_{\text {tpa }} / \mathrm{nm}$ & $\sigma_{\mathrm{tpa}} / \mathrm{GM}$ & Molecule & $E_{\text {tpa }} / \mathrm{eV}$ & $\lambda_{\text {tpa }} / \mathrm{nm}$ & $\sigma_{\text {tpa }} / \mathrm{GM}$ \\
\hline \multirow[t]{9}{*}{$\mathrm{HCH}$} & 2.32 & 1066 & 864 & $\mathrm{HCH}-\mathrm{CNO}$ & 2.41 & 1026 & 856 \\
\hline & 2.83 & 874 & 66 & & 2.89 & 856 & 66 \\
\hline & 3.38 & 732 & 762 & & 3.46 & 715 & 617 \\
\hline & 3.69 & 670 & 23 & & 3.78 & 654 & 173 \\
\hline & 3.76 & 658 & 138 & & 3.79 & 653 & 0 \\
\hline & 4.00 & 618 & 1421 & & 3.81 & 649 & 201 \\
\hline & 4.10 & 603 & 1306 & & 4.08 & 606 & 453 \\
\hline & 4.26 & 581 & 17 & & 4.12 & 600 & 2824 \\
\hline & & & & & & $860^{*}$ & $1642^{*}$ \\
\hline \multirow[t]{9}{*}{$\mathrm{HCM}$} & 2.29 & 1080 & 807 & HCM-CNO & 2.39 & 1035 & 808 \\
\hline & 2.80 & 883 & 68 & & 2.86 & 865 & 68 \\
\hline & 3.35 & 738 & 729 & & 3.43 & 721 & 601 \\
\hline & 3.64 & 679 & 31 & & 3.74 & 661 & 74 \\
\hline & 3.76 & 658 & 114 & & 3.75 & 659 & 0 \\
\hline & 3.98 & 621 & 1207 & & 3.80 & 651 & 252 \\
\hline & 4.07 & 608 & 1044 & & 4.08 & 606 & 235 \\
\hline & 4.21 & 587 & 39 & & 4.10 & 603 & 2427 \\
\hline & & & & & & $860^{*}$ & $980^{*}$ \\
\hline
\end{tabular}


表 5 分子在水中最低八个激发态的激发能、对应的双光子吸收波长以及双光子吸收截面

Table 5 Excitation energy, the corresponding two-photon absorption wavelength, and the TPA cross section of the lowest eight excited states for the compounds in $\mathrm{H}_{2} \mathrm{O}$

\begin{tabular}{|c|c|c|c|c|c|c|c|}
\hline Molecule & $E_{\mathrm{tp} a} / \mathrm{eV}$ & $\lambda_{\mathrm{p} a \mathrm{a}} / \mathrm{nm}$ & $\sigma_{\mathrm{tpp}} / \mathrm{GM}$ & Molecule & $E_{\mathrm{tp} a} / \mathrm{eV}$ & $\lambda_{\text {tpa }} / \mathrm{nm}$ & $\sigma_{\mathrm{tpa}} / \mathrm{GM}$ \\
\hline \multirow[t]{9}{*}{$\mathrm{HCH}$} & 2.32 & 1066 & 872 & $\mathrm{HCH}-\mathrm{CNO}$ & 2.42 & 1022 & 867 \\
\hline & 2.83 & 874 & 65 & & 2.90 & 853 & 65 \\
\hline & 3.39 & 730 & 775 & & 3.47 & 713 & 625 \\
\hline & 3.70 & 668 & 26 & & 3.79 & 653 & 224 \\
\hline & 3.76 & 658 & 133 & & 3.81 & 649 & 0 \\
\hline & 3.97 & 623 & 1479 & & 3.81 & 649 & 160 \\
\hline & 4.10 & 603 & 1355 & & 4.08 & 606 & 575 \\
\hline & 4.27 & 579 & 18 & & 4.13 & 599 & 2825 \\
\hline & & & & & & $860^{*}$ & $1642^{*}$ \\
\hline \multirow[t]{9}{*}{$\mathrm{HCM}$} & 2.30 & 1075 & 822 & $\mathrm{HCM}-\mathrm{CNO}$ & 2.39 & 1035 & 816 \\
\hline & 2.80 & 883 & 67 & & 2.87 & 862 & 68 \\
\hline & 3.36 & 736 & 741 & & 3.44 & 719 & 611 \\
\hline & 3.65 & 678 & 33 & & 3.75 & 659 & 84 \\
\hline & 3.76 & 658 & 111 & & 3.76 & 658 & 0 \\
\hline & 3.98 & 621 & 1265 & & 3.81 & 649 & 252 \\
\hline & 4.08 & 606 & 1086 & & 4.08 & 606 & 281 \\
\hline & 4.22 & 586 & 39 & & 4.11 & 602 & 2501 \\
\hline & & & & & & $860^{*}$ & $980^{*}$ \\
\hline
\end{tabular}

*experimental results ${ }^{19}\left(1 \mathrm{GM}=10^{-50} \mathrm{~cm}^{4} \cdot \mathrm{s} \cdot\right.$ photon $\left.^{-1}\right)$

大双光子吸收截面增加到 2825 和 $2501 \mathrm{GM}$, 对应 的吸收波长分别蓝移到 599、602 $\mathrm{nm}$ 处。其中, $\mathrm{HCH}$ 和 $\mathrm{HCM}$ 分子在水溶液中的最大双光子吸收截 面比在 DMSO 溶液中的最大双光子吸收截面均增 大了 $58 \mathrm{GM}$, 这是由于溶剂效应导致的。在同种 溶剂中, $\mathrm{HCH}$ 分子的最大双光子吸收截面大于 $\mathrm{HCM}$ 分子的最大截面, 可见末端烷基长度的增加 可以提高分子的双光子吸收截面, 这与实验测量 结果在趋势上是吻合的。而与实验测量得到的 $\mathrm{HCH}-\mathrm{CNO}$ 和 HCM-CNO 分子的最大双光子吸收截 面 1642、980 GM 相比较, 理论计算的数值偏大, 这主要是由于目前的理论研究忽略了光场和分子间 相互作用及分子的振动对双光子吸收截面的影响。

以上计算结果表明，两探针分子 $\mathrm{HCH}$ 和 $\mathrm{HCM}$ 均具有较大的双光子吸收截面, 并且与 $\mathrm{ClO}^{-}$反应 后, 分子的最大双光子吸收截面有不同程度的增 大，这意味着两分子均可作为较好的双光子苂光 $\mathrm{ClO}^{-}$探针, 此外以已基作为吡啶所连的末端基团 的 $\mathrm{HCH}$ 分子具有更大的双光子吸收截面，并且与 $\mathrm{ClO}^{-}$反应后分子的双光子吸收能力增强更为明 显, 因此可以预测该分子可作为性能更优的双光 子苂光探针。

\section{4 荧光性质}

$\mathrm{Li}$ 等 ${ }^{19}$ 在实验上给出了探针分子探测前后的发
射波长变化情况, 结果表明随着 $\mathrm{ClO}^{-}$的不断滴 定, 含有 $\mathrm{HCH}$ 分子和 $\mathrm{HCM}$ 分子的溶液分别在 546、544 nm 处展现出增强的苂光发射峰。理论计 算得到的分子发射能量、对应的苂光波长、振子 强度、跃迁特性以及由 Einstein 跃迁几率公式 ${ }^{23}$ 得 到的苂光寿命在表 6 中列出。可以看到, $\mathrm{HCH}$ 、 $\mathrm{HCM}$ 分子在水溶液和在 DMSO 溶液中的苂光发射 峰位分别位于 589、592 nm 处和 593、596 nm 处, 对应的荧光发射强度分别为 $0.59 、 0.56$ a.u. 和 0.57 、 0.54 a.u., 与 $\mathrm{ClO}^{-}$反应后, $\mathrm{HCH}-\mathrm{CNO} 、 \mathrm{HCM}-\mathrm{CNO}$ 分子在水溶液和在 DMSO 溶液中的苂光发射峰位 分别蓝移到了550、553 nm 处和 553、557 nm 处, 对应的苂光发射强度增强到了 $0.77 、 0.72$ a.u. 和 0.73、0.68 a.u.。 $\mathrm{ClO}^{-}$的加入使得体系苂光强度增 强, 这与实验结果定性一致, 说明这两分子均可 作为性能优良的菼光探针。

荧光寿命是衡量探针性能的重要参数, 与反 应前的分子体系 $\mathrm{HCH}$ 和 $\mathrm{HCM}$ 分子相比, $\mathrm{HCH}$ $\mathrm{CNO}$ 和 $\mathrm{HCM}-\mathrm{CNO}$ 分子在水溶液和在 DMSO 溶液 中的苂光寿命均发生了不同程度的缩短，因此与 探测底物反应以后, 分子发出的荧光信号更容易 被探测到, 这同样证明了两分子均可作为性能优 异的荧光探针。另外, $\mathrm{HCH}$ 和 $\mathrm{HCH}-\mathrm{CNO}$ 分子具 有比 HCM 和 HCM-CNO 分子更短的菼光寿命, 因 
表 6 分子在低能量范围内的发射性质，包括发射能 $\left(\boldsymbol{E}_{\mathrm{ope}}\right)$ 、对应的发射波长 $\left(\lambda_{\mathrm{op}}\right)$ 、振子强度 $\left(\boldsymbol{\delta}_{\mathrm{ope}}\right)$ 、跃迁特性和荧光辐射寿命 $(\tau)$

Table 6 Emission properties of the compounds in lower energy region, including the emission energy $\left(E_{\text {ope }}\right)$, the corresponding emission wavelength $\left(\lambda_{\text {ope }}\right)$, the oscillator strength $\left(\delta_{\text {ope }}\right)$, the transition feature and the emission lifetime $(\tau)$

\begin{tabular}{|c|c|c|c|c|c|}
\hline Molecule & $E_{\mathrm{opc}} / \mathrm{eV}$ & $\lambda_{\text {ope }} / \mathrm{nm}$ & $\delta_{\text {oped }} /$ (a.u.) & Transition feature & $\tau / \mathrm{ns}$ \\
\hline \multirow[t]{3}{*}{$\mathrm{HCH}$} & 2.10 & $589^{\mathrm{a}}$ & 0.59 & $S_{1} \rightarrow S_{0}, \mathrm{H} \rightarrow \mathrm{L}, 99.5 \%$ & 8.87 \\
\hline & 2.09 & $593^{\mathrm{b}}$ & 0.57 & $S_{1} \rightarrow S_{0}, \mathrm{H} \rightarrow \mathrm{L}, 99.5 \%$ & 9.27 \\
\hline & & $546^{\mathrm{c}}$ & & & \\
\hline \multirow[t]{3}{*}{$\mathrm{HCH}-\mathrm{CNO}$} & 2.25 & $550^{\mathrm{a}}$ & 0.77 & $S_{1} \rightarrow S_{0}, \mathrm{H} \rightarrow \mathrm{L}, 99.3 \%$ & 5.92 \\
\hline & 2.24 & $553^{\mathrm{b}}$ & 0.73 & $S_{1} \rightarrow S_{0}, \mathrm{H} \rightarrow \mathrm{L}, 99.3 \%$ & 6.30 \\
\hline & & $546^{\mathrm{c}}$ & & & \\
\hline \multirow[t]{3}{*}{$\mathrm{HCM}$} & 2.09 & $592^{\mathrm{a}}$ & 0.56 & $S_{1} \rightarrow S_{0}, \mathrm{H} \rightarrow \mathrm{L}, 99.5 \%$ & 9.43 \\
\hline & 2.08 & $596^{\mathrm{b}}$ & 0.54 & $S_{1} \rightarrow S_{0}, \mathrm{H} \rightarrow \mathrm{L}, 99.5 \%$ & 9.88 \\
\hline & & $544^{\mathrm{c}}$ & & & \\
\hline \multirow[t]{3}{*}{$\mathrm{HCM}-\mathrm{CNO}$} & 2.24 & $553^{\mathrm{a}}$ & 0.72 & $S_{1} \rightarrow S_{0}, \mathrm{H} \rightarrow \mathrm{L}, 99.3 \%$ & 6.39 \\
\hline & 2.23 & $557^{\mathrm{b}}$ & 0.68 & $S_{1} \rightarrow S_{0}, \mathrm{H} \rightarrow \mathrm{L}, 99.4 \%$ & 6.82 \\
\hline & & $544^{\mathrm{c}}$ & & & \\
\hline
\end{tabular}

${ }^{\mathrm{a}}$ in $\mathrm{H}_{2} \mathrm{O}$, ${ }^{\mathrm{b}}$ in DMSO, ${ }^{\mathrm{c}}$ experimental results ${ }^{19}$

此 $\mathrm{HCH}$ 分子可作为性能更优的苂光探针分子。

\section{5 响应机理}

分子体系的光学性质强烈依赖于电荷分布情 况, 因此为了探究探针分子的响应机理, 我们进一 步理论计算了分子在基态和电荷转移态的 Mulliken 电荷分布以及在两态之间的电荷转移情况, 为了 更清晰地展示结果, 我们将所研究的分子体系分 为三个部分 (见图 1)。 $\mathrm{A}$ 部分和 $\mathrm{C}$ 部分为分子的末 端基团, $\mathrm{B}$ 部分为分子共轭桥部分。表 7 列出了各 部分基态和电荷转移态的 Mulliken 电荷分布以及在 两态之间的电荷转移情况。由表中数据可以看 到, 在基态时, $\mathrm{HCH}$ 分子和 $\mathrm{HCM}$ 分子的 $\mathrm{A} 、 \mathrm{~B} 、 \mathrm{C}$ 部分的电荷量均为 $0.917 e 、 0.112 e 、-0.029 e$, 与 $\mathrm{ClO}^{-}$反应后, 生成物 $\mathrm{HCH}-\mathrm{CNO}$ 分子和 $\mathrm{HCM}-\mathrm{CNO}$ 分子 $\mathrm{A} 、 \mathrm{~B} 、 \mathrm{C}$ 部分的电荷量分别为 0.927 、 $0.318 e 、-0.245 e$ 和 $0.926 e 、 0.318 e 、-0.244 e$, 可 见 $\mathrm{ClO}^{-}$的加入使得识别基团肜中的碳氮双键被氧 化为碳氮三键, 末端基团由 $-\mathrm{OH}$ 变为 $-\mathrm{O}^{-}$, 进一 步影响了分子体系的电荷分布和光学性质。为了
直观地看到结合 $\mathrm{ClO}^{-}$对两探针分子电荷分布的影 响, 我们做出了分子的 MEPs。如图 2 所示, 与 $\mathrm{ClO}^{-}$反应后, $\mathrm{HCH}$ 分子和 $\mathrm{HCM}$ 分子 $\mathrm{C}$ 部分的负电 荷密度稍有增大，且两探针分子在反应前后的正 电荷密度都主要分布在 $\mathrm{A}$ 部分。对于两探针分子 $\mathrm{HCH}$ 和 $\mathrm{HCM}$, 电荷分布的变化是由分子末端基团 中 $\mathrm{C}=\mathrm{N}$ 键的结构变化引起的。之前的一些报道发 现, 当化合物分子中含有 $\mathrm{C}=\mathrm{N}$ 键时, $\mathrm{C}=\mathrm{N}$ 异构 化会加剧分子的激发态的非辐射跃迁，成为激发 态能量主要的衰减途径, 从而使得分子苂光淬 灭; 当通过桥键将 $\mathrm{C}=\mathrm{N}$ 键固定以后, $\mathrm{C}=\mathrm{N}$ 异构 化会受到抑制, 从而使得分子的苂光恢复 ${ }^{24-27}$ 。因 此该系列探针分子的响应机理可归于 $\mathrm{ClO}^{-}$的加入 使得 $\mathrm{C}=\mathrm{N}$ 异构化被抑制, 进而导致了苂光信号的 增强。

第一激发态构型下，探针分子 $\mathrm{HCH}$ 和 $\mathrm{HCM}$ 中 $\mathrm{A} 、 \mathrm{C}$ 部分的电荷量较基态构型下的电荷量增加到 $0.784 e 、-0.103 e$ 和 $0.783 e 、-0.103 e$, 而 $\mathrm{B}$ 部分的 电荷量分别减小到 $0.319 e 、 0.320 e$, 因而 $\mathrm{A} 、 \mathrm{C}$ 部

表 7 分子在基态和电荷转移态下 A、B、C 三部分的 Mulliken 电荷量以及两态之间的电荷转移量

Table 7 Mulliken charges of groups A, B and C of compounds for the charge-transfer state and ground state and their charge difference

\begin{tabular}{ccccccrrrr}
\hline Molecule & & $\mathrm{A}$ & $\mathrm{B}$ & $\mathrm{C}$ & Molecule & & $\mathrm{A}$ & $\mathrm{B}$ & $\mathrm{C}$ \\
\hline $\mathrm{HCH}$ & $S_{0}$ & 0.917 & 0.112 & -0.029 & HCH-CNO & $S_{0}$ & 0.927 & 0.318 & -0.245 \\
& $S_{1}$ & 0.784 & 0.319 & -0.103 & & $S_{1}$ & 0.789 & 0.480 & -0.269 \\
& $\Delta Q$ & -0.133 & 0.207 & -0.074 & & $\Delta Q$ & -0.138 & 0.162 & -0.024 \\
\multirow{3}{*}{$\mathrm{HCM}$} & $S_{0}$ & 0.917 & 0.112 & -0.029 & HCM-CNO & $S_{0}$ & 0.926 & 0.318 & -0.244 \\
& $S_{1}$ & 0.783 & 0.320 & -0.103 & & $S_{1}$ & 0.788 & 0.481 & -0.269 \\
& $\Delta Q$ & -0.134 & 0.208 & -0.074 & & $\Delta Q$ & -0.138 & 0.163 & -0.025 \\
\hline
\end{tabular}



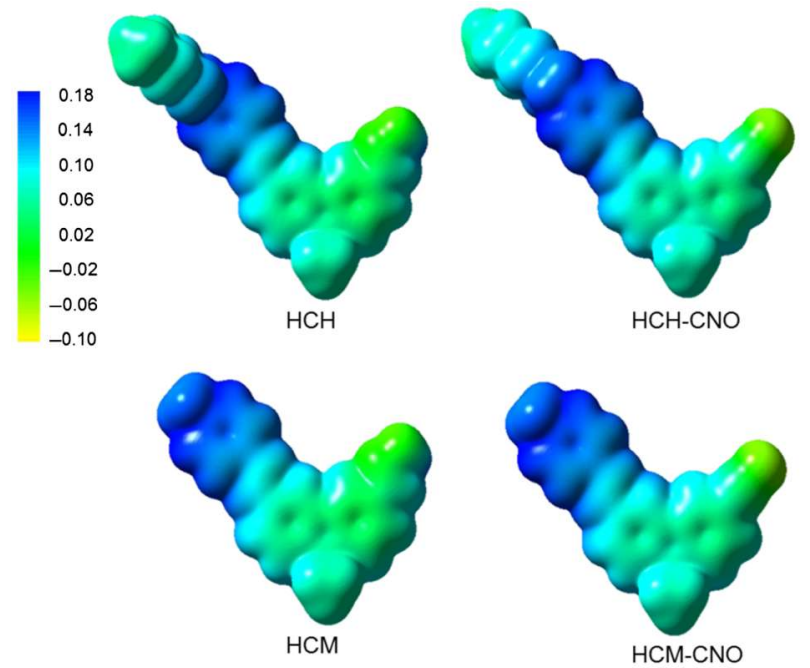

图 2 分子的静电势图

Fig.2 Diagram of molecular electrostatic potential

分均表现出得电子的能力, 而 $\mathrm{B}$ 部分表现出失电子 能力。与 $\mathrm{ClO}^{-}$反应后, 生成物 $\mathrm{HCH}-\mathrm{CNO}$ 和 $\mathrm{HCM}-$ $\mathrm{CNO}$ 在第一激发态构型下 $\mathrm{A} 、 \mathrm{~B} 、 \mathrm{C}$ 基团电荷量分 别为 $0.789 e 、 0.480 e 、-0.269 e$ 和 $0.788 e 、 0.481 e$ 、 $-0.269 e$ 。需要注意的是, 探针分子与 $\mathrm{ClO}^{-}$反应前 后从基态到第一激发态的电荷转移量发生了较大 变化。与探针分子 $\mathrm{HCH}$ 相比, $\mathrm{HCH}-\mathrm{CNO}$ 分子 $\mathrm{B}$ 基团的供电荷量由 $0.207 e$ 减小到 $0.162 e, \mathrm{C}$ 基团的 得电荷量由 $0.074 e$ 减小到 $0.024 e$ 。与探针分子 $\mathrm{HCM}$ 相比, $\mathrm{HCM}-\mathrm{CNO}$ 分子 $\mathrm{B}$ 基团的供电荷量由 $0.208 e$ 减小到 $0.163 e, \mathrm{C}$ 基团的得电荷量由 $0.074 e$ 减小到 $0.025 e$ 。以上结果表明, $\mathrm{ClO}^{-}$的加入使得肪 基团中的碳氮双键被氧化为碳氮三键，同时 $-\mathrm{OH}$ 变为 $-\mathrm{O}^{-}$, 因而改变了体系激发过程中的电荷转 移情况。

\section{4 结 论}

研究了实验室新合成的水溶性 $\mathrm{ClO}^{-}$双光子苂 光探针分子 $\mathrm{HCH}$ 和 $\mathrm{HCM}$ 的单、双光子吸收以及苂 光发射性质, 着重讨论了 $\mathrm{ClO}^{-}$的加入对探针分子 光学性质的影响, 并利用电荷布居探究了两探针 分子的响应机理。计算结果表明, 在低能量范围 内, $\mathrm{HCH}$ 分子和 $\mathrm{HCM}$ 分子在与 $\mathrm{ClO}^{-}$反应前后的最 大单光子吸收态均发生在第一激发态, 反应后对 应的最大单光子吸收峰位和最大双光子吸收峰位 均发生了明显的蓝移。另外, 两探针分子与 $\mathrm{ClO}$ 作用后，对应的苂光发射强度展现出不同程度的 增强, 这与实验测量结果吻合较好。两分子均可
作为较好的 $\mathrm{ClO}^{-}$双光子荧光探针, 由于分子 $\mathrm{HCH}$ 在与 $\mathrm{ClO}^{-}$反应前后具有更强的光吸收和光发射以 及更为明显的荧光增强因而可作为更优秀的双光 子苂光探针。通过分析探针分子 $\mathrm{HCH}$ 和 $\mathrm{HCM}$ 的电 荷布居情况, 表明 $\mathrm{ClO}^{-}$的加入使得分子体系的 $\mathrm{C}=\mathrm{N}$ 异构化被抑制, 进而改变了分子体系的电荷 分布和光学性质。我们的研究对于理解探针分子 的响应机理以及分子结构和光学性质之间的关 系, 帮助实验合成性能良好的双光子荧光探针具 有一定的指导作用。

\section{References}

(1) Gomes, A.; Fernandes, E.; Lima, J. L. F. C. J. Biochem. Biophys. Methods 2005, 65 (2), 45. doi: 10.1016/j. jbbm.2005.10.003

(2) Fink, M. P. Curr. Opin. Crit. Care. 2002, 8 (1), 6. doi:10.1097/ 00075198-200202000-00002

(3) Li, X. H.; Zhang, G. X.; Ma, H. M.; Zhang, D. Q.; Li, J.; Zhu, D. B. J. Am. Chem. Soc. 2004, 126 (37), 11543. doi: 10.1021/ ja0481530

(4) Cui, L. L.; Zhou, D. H.; Li, M. M. Acta Phys. -Chim. Sin. 2013, 29 (4), 745. [崔俐丽, 周丹红, 李苗苗. 物理化学学报, 2013, 29 (4), 745.] doi: 10.3866/PKU.WHXB201302064

(5) Yuan, L.; Wang, L.; Agrawalla, B. K.; Park. S. J.; Zhu, H.; Sivaraman, B.; Peng, J. J.; Xu, Q. H.; Chang, Y. T. J. Am. Chem. Soc. 2015, 137, 5930. doi: 10.1021/jacs.5b00042

(6) Xu, Q.; Heo, C. H.; Kim, G.; Lee, H. W.; Kim, H. M.; Yoon, J. Angew. Chem. Int. Ed. 2015, 54, 4890. doi: 10.1002/ anie. 201500537

(7) Pattison, D. I.; Davies, M. J. Chem. Res. Toxicol. 2001, 14 (10), 1453. doi: $10.1021 / \mathrm{tx} 0155451$

(8) O'Brien, P. J. Chem. Biol. Inter. 2000, 129 (2), 113. doi: 10.1016/S0009-2797(00)00201-5

(9) Podrez, E. A.; Abu-Soud, H. M.; Hazen, S. L. Free Radical Biol. Med. 2000, 28 (12), 1717. doi: 10.1016/S0891-5849(00)00229-X

(10) Cheng, G. H.; Fan, J. L.; Sun, W.; Sui, K.; Jin, X.; Wang, J. Y.; Peng, X. J. Analyst 2013, 138, 6091. doi: 10.1039/c3an01152f

(11) Xiao, H. D.; Xin, K.; Dou, H. F.; Yin, G.; Quan, Y. W.; Wang, R. Y. Chem. Commun. 2015, 51, 1442. doi: 10.1039/c4cc07411d

(12) Setsukinai, K.; Urano, Y.; Kakinuma, K.; Majima, H. J.; Nagano, T. J. Biol. Chem. 2003, 278 (5), 3170. doi: 10.1074/jbc. M209264200

(13) Takats, Z.; Koch, K. J.; Cooks, R. G. Analyt. Chem. 2001, 73 (18), 4522. doi: 10.1021/ac010338r

(14) Yang, W. J.; Zhang, Y. J.; Wang, C. K. Acta Phys. -Chim. Sin. 2015, 31 (12), 2303. [杨文静, 张玉瑾, 王传奎. 物理化学学报, 2015, 31 (12), 2303.] doi: 10.3866/PKU.WHXB201510233

(15) Zhou, D. H.; Li, M. M.; Cui, L. L. Acta Phys. -Chim. Sin. 2013, 29 (7), 1453. [周丹红, 李苗苗, 崔俐丽. 物理化学学报, 2013, 
29 (7), 1453.] doi: 10.3866/PKU.WHXB201304244

(16) Meng, X. M.; Wang, S. X.; Li, Y. M.; Zhu, M. Z.; Guo, Q. X. Chem. Commun. 2012, 48, 4196. doi: 10.1039/c2cc30471f

(17) Yin, H. J.; Zhang, B. C.; Yu, H. Z.; Zhu, L.; Feng, Y.; Zhu, M. Z.; Guo, Q. X.; Meng, X. M. J. Org. Chem. 2015, 80, 4306. doi: $10.1021 /$ jo502775t

(18) Ning, P.; Jiang, J. C.; Li, L. C.; Wang, S. X.; Yu, H. Z.; Feng, Y.; Zhu, M. Z.; Zhang, B. C.; Yin, H.; Guo, Q. X.; Meng, X. M. Biosens. Bioelectron. 2016, 77, 921. doi: 10.1016/j. bios.2015.10.061

(19) Li, D. X.; Feng, Y.; Lin, J. Z.; Chen, M.; Wang, S. X.; Wang, X.; Sheng, H. T.; Shao, Z. L.; Zhu, M. Z.; Meng, X. M. Sens. Actuators B 2016, 222, 483. doi: 10.1016/j.snb.2015.08.098

(20) Ding, H. J.; Sun, J.; Zhang, Y. J.; Wang, C. K. Chem. Phys. Lett. 2014, 591, 142. doi: 10.1016/j.cplett.2013.11.015

(21) Frisch, M. J.; Trucks, G. W.; Schlegel, H. B.; et al. Gaussian 09, Revision A.01; Gaussian Inc.: Wallingford, CT, 2009.
(22) Aidas, K.; Angeli, C.; Bak, K. L.; et al. WIREs Comput. Mol. Sci. 2014, 4, 269. doi: 10.1002/wcms.2014.4.issue-3

(23) Zhang, Y. J.; Zhang, Q. Y.; Ding, H. J.; Song, X. N.; Wang, C. K. Chin. Phys. B 2015, 24 (2), 023301. doi: 10.1088/1674-1056/ 24/2/023301

(24) Wang, X. M.; Wang, X. H.; Feng, Y.; Zhu, M. Z.; Yin, H.; Guo, Q. X.; Meng, X. M. Dalton Trans. 2015, 44 (14), 6613. doi: $10.1039 / \mathrm{c} 5 \mathrm{dt} 00012 \mathrm{~b}$

(25) Wu, J. S.; Liu, W. M.; Zhuang, X. Q.; Wang, F.; Wang, P. F.; Tao, S. L.; Zhang, X. H.; Wu, S. K.; Lee, S. T. Org. Lett. 2007, 9 (1), 33. doi: 10.1021/ol062518z

(26) Liu, Z. D.; Xu, H. J.; Chen, S. S.; Sheng, L. Q.; Zhang, H.; Hao, F. Y.; Su, P. F.; Wang, W. L. Spectrochim. Acta A 2015, 149, 8. doi: 10.1016/j.saa.2015.04.030

(27) Torawane, P.; Tayade, K.; Bothra, S.; Sahoo, S. K.; Singh, N.; Borse, A.; Kuwar, A. Sens. Actuators B 2016, 222, 562. doi: 10.1016/j.snb.2015.08.104 